\title{
Pre-hospital stroke screening and notification of patients with reperfusion-eligible acute ischaemic stroke using modified Face Arm Speech Time test
}

\author{
William CY Leung, Kay C Teo, WM Kwok, Lawrence HC Lam, Olivia MY Choi, Mona MY Tse, WM Lui, \\ TC Tsang, Anderson CO Tsang *
}

\section{A B S T R A C T}

Objectives: To investigate the effects of pre-hospital stroke screening and notification on reperfusion therapy for patients with acute ischaemic stroke.

Methods: Pre-hospital stroke screening criteria were established based on a modified version of the Face Arm Speech Time (FAST) test. Screening was performed during ambulance transport by emergency medical service (EMS) personnel who completed a 2-hour training session on stroke screening. Temporal trends affecting acute ischaemic stroke investigation and intervention were compared before and after implementation of the pre-hospital screening.

Results: From July 2018 to October 2019, 298 patients with suspected stroke were screened by EMS personnel during ambulance transport prior to hospital arrival. Of these 298 patients, 213 fulfilled the screening criteria, 166 were diagnosed with acute stroke, and 32 received reperfusion therapy. The onset-to-door time was shortened by more than 1.5 hours $(100.6 \mathrm{~min}$ vs $197.6 \mathrm{~min}, \mathrm{P}<0.001)$. The door-to-computed tomography time (25.6 min vs $32.0 \mathrm{~min}, \mathrm{P}=0.021)$, door-to-needle time (49.2 $\mathrm{min}$ vs $70.1 \mathrm{~min}, \mathrm{P}=0.003)$, and door-to-groin puncture time for intra-arterial mechanical thrombectomy (126.7 min vs $168.6 \mathrm{~min}, \mathrm{P}=0.04$ ) were significantly shortened after implementation of the pre-hospital screening and notification, compared with historical control data of patients admitted from January 2018 to June 2018, before implementation of the screening system.

Conclusion: Implementation of pre-hospital stroke screening using criteria based on a modified version of the FAST test, together with pre-arrival notification, significantly shortened the door-to-reperfusion therapy time for patients with ischaemic stroke. Pre-hospital stroke screening during ambulance transport by EMS personnel who complete a 2-hour focused training session is effective for identifying reperfusion-eligible patients with stroke.

\section{Hong Kong Med J 2020;26:479-85}

https://doi.org/10.12809/hkmj208552

\author{
${ }^{1}$ WCY Leung, MB, BS \\ ${ }^{1} \mathrm{KC}$ Teo, $\mathrm{MB}, \mathrm{BS}$ \\ ${ }^{2}$ WM Kwok, MB, BS \\ ${ }^{3}$ LHC Lam, MSC \\ ${ }^{4}$ OMY Choi, MPsych \\ ${ }^{1}$ MMY Tse, MB, BS \\ ${ }^{4}$ WM Lui, MB, BS \\ TC Tsang, $M B, B S$ \\ ${ }^{4}$ ACO Tsang *, MB, BS
}

${ }^{1}$ Division of Neurology, Department of Medicine, Queen Mary Hospital, The University of Hong Kong, Hong Kong

2 Department of Accident and Emergency, Queen Mary Hospital, Hong Kong

Hong Kong Division, Ambulance Command, Hong Kong Fire Services Department, Hong Kong

${ }^{4}$ Division of Neurosurgery, Department of Surgery, Queen Mary Hospital,

The University of Hong Kong, Hong Kong

* Corresponding author: acotsang@hku.hk

New knowledge added by this study

- Implementation of pre-hospital stroke screening using criteria based on a modified version of the Face Arm Speech Time (FAST) test, together with pre-arrival notification, significantly shortened the door-toreperfusion therapy time for patients with acute ischaemic stroke.

- The onset-to-door time, door-to-computed tomography time, door-to-needle time, and door-to-groin puncture time for intra-arterial mechanical thrombectomy were all significantly shortened after implementation of the pre-hospital screening and notification, compared with historical control data.

- Pre-hospital stroke screening during ambulance transport by emergency medical service personnel who complete a 2-hour focused training session is effective for identifying patients with reperfusion-eligible stroke.

Implications for clinical practice or policy

- The findings confirm the importance of pre-hospital notification in facilitating downstream management of patients with acute stroke by allowing the Accident and Emergency Department and stroke team to prepare for the patient's arrival.

- Cost-effectiveness studies are needed to evaluate the impact and sustainability of the system on a territory-wide scale, which will aid in long-term infrastructure development in acute stroke care. 


\section{基於中風「快速」測試（FAST）修改版標準對 符合再灌注治療的急性缺血性中風患者進行院前 中風篩查和通報}

\author{
梁俊彥、張記暢、郭詠文、林浩璋、蔡明欣、謝曼瑜、 \\ 呂偉文、曾達之、曾震安 \\ 目的：探討急性缺血性中風患者院前中風篩查和通報再灌注治療的影 \\ 響。 \\ 方法：基於中風「快速」測試（FAST）修改版建立院前中風篩查標 \\ 準。已完成2小時中風篩查培訓的緊急醫療服務人員在救護車運輸過 \\ 程中為患者進行院前篩查。將院前中風篩查實施前和實施後對影響急 \\ 性缺血性中風檢查和干預的時間趨勢作出比較。 \\ 結果：2018年7月至2019年10月，緊急醫療服務人員在救護車運 \\ 輸過程中對298名疑似中風患者進行院前篩查。這298例患者中, \\ 213 例符合篩查標準, 其中 166 例被診斷為急性中風, 其中32例接受 \\ 再灌注治療。與2018年1月至2018年6月入院患者的歷史對照數據, \\ 即實施院前中風篩查前相比, 實施院前中風篩查後發病至入院需時減 \\ 少超過 1.5 小時 $(100.6$ 分鐘比 197.6 分鐘, $\mathrm{P}<0.001)$ 。入院至電腦 \\ 掃描 (25.6分鐘比 32.0 分鐘, $\mathrm{P}=0.021)$ 、入院至上針 (49.2分鐘比 \\ 70.1 分鐘, $\mathrm{P}=0.003)$ ，以及入院至股動脈穿刺進行機械取栓術 \\ (126.7 分鐘比 168.6 分鐘, $\mathrm{P}=0.04)$ 需時也明顯縮短。 \\ 結論：使用基於中風「快速」測試（FAST）修改版建立院前中風篩查 \\ 標準並結合入院前通報, 可顯著縮短缺血性中風患者的入院至再灌注 \\ 治療時間。由完成2小時中風篩查培訓的緊急醫療服務人員在救護車 \\ 運輸過程中為患者進行院前穊查, 能有效識別符合再灌注治療條件的 \\ 中風患者。
}

recent recommendation for establishing a system of care for patients with stroke. ${ }^{2,3}$

Before 2018, there was no pre-hospital stroke notification system in Hong Kong. ${ }^{4}$ Patients with ischaemic stroke, including those who are within the therapeutic window for reperfusion therapy, are transferred to the nearest AED under the regular triage system, which may delay reperfusion treatment. Herein, we investigate the effects of a team-based pre-hospital stroke notification system designed to improve the delivery of acute stroke care.

\section{Methods}

\section{Pre-hospital stroke notification system}

Our pre-hospital stroke notification system was established in collaboration between the AED, stroke neurologists, neurosurgeons, and the ambulance service. This included an advance hospital notification by EMS personnel, rapid triage protocol, advance stroke team notification, and image acquisition via CT. The EMS personnel screened patients with suspected stroke using a locally formulated pre-hospital stroke assessment scale to identify reperfusion-eligible patients with stroke. For patients who met all criteria, advance hospital notification was activated by calling a designated number in the AED; this call served to alert the stroke team, triage station, and on-duty AED medical officer. A CT scan was then arranged by the AED medical officer in the next available urgent slot.

\section{Introduction}

Timely reperfusion for ischaemic stroke with intravenous thrombolysis (IVT) or mechanical thrombectomy can substantially improve patient outcomes. ${ }^{1}$ Hence, all possible efforts should be made to shorten the stroke onset-to-treatment time. The major benchmark for measurement of acute stroke treatment speed is the door-to-needle time, that is, the duration between Accident and Emergency Department (AED) arrival and administration of IVT. Other benchmarks include duration between AED arrival and brain computed tomography (doorto-CT time) and duration between AED arrival and groin puncture for mechanical thrombectomy (door-to-groin puncture time). The recommended intervals are within 60 minutes for door-toneedle time, 25 minutes for door-to-CT time, and 120 minutes for door-to-groin puncture time.

The American Heart Association/American Stroke Association Target Stroke initiative supports implementation of strategies to shorten the doorto-needle time. These include advance hospital notification by emergency medical service (EMS) personnel, rapid triage protocol in the AED, rapid acquisition of brain imaging data, and a team-based approach. These strategies were consolidated in the

\section{Ambulance stroke assessment}

Our simple pre-hospital stroke assessment in Chinese was formulated on the basis of the Los Angeles Pre-hospital Stroke Screen and the Face drooping, Arm weakness, Speech difficulty, Time to call 911 (FAST) screening criteria. ${ }^{5,6}$ In contrast to the Los Angeles Pre-hospital Stroke Screen and FAST criteria, we aimed to identify only patients eligible for reperfusion therapy, in whom earlier treatment would provide the greatest benefit. The inclusion criteria were as follows: $(1) \leq 4$ hours since onset of symptoms, (2) sudden limb weakness or speech impairment, (3) age >18 years, (4) stroke unrelated to recent trauma, (5) Glasgow Coma Scale score $\geq 8$, (6) systolic blood pressure of $>100 \mathrm{~mm} \mathrm{Hg}$, (7) no history of seizures/epilepsy, and (8) absence of previous wheelchair-bound or bed-ridden status. Patients who satisfied all eight inclusion criteria underwent a point-based Chinese FAST screening (speech disturbance $=2$ points; unilateral facial drooping = 1 point; and unilateral limb weakness = 2 points). Advance hospital notification was activated for eligible patients with $\geq 2$ points in the FAST screening. 


\section{Emergency medical service personnel training}

All EMS personnel within our hospital's catchment area attended a 2-hour stroke training session delivered by a team of stroke neurologists, neurosurgeons, and emergency physicians. This included didactic instruction concerning stroke subtypes, symptoms, reperfusion therapy prehospital management, and hands-on training for utilisation of the ambulance stroke assessment scale.

\section{Patients}

The pre-hospital stroke screening and notification system was implemented on 1 July 2018. All patients with acute stroke admitted via ambulance transfer from July 2018 to October 2019 were prospectively included in this study. Data were collected concerning patient characteristics, stroke severity, and temporal trends of stroke treatment. Patients admitted via the AED whose admission had included acute stroke diagnosis, during the period from January 2018 to June 2018 (6 months before implementation of the pre-hospital stroke screening and notification system), were retrospectively identified as historical controls.

\section{Statistical analysis}

Categorical variables were compared using Chi squared analysis, while continuous variables were compared using independent $t$ tests and one-way analysis of variance. Univariate analysis was initially used on all variables; the results were recorded as odds ratios with 95\% confidence intervals. KaplanMeier curve and log rank test analyses of door-toneedle time with and without notification were performed. Statistical significance was set at $\mathrm{P}<0.05$. All analyses were performed using SPSS Statistics for Windows, version 25.0 (IBM Corp, Armonk [NY], United States).

\section{Results}

During the study period, 298 patients with suspected stroke were screened by the ambulance staff. Of these 298 patients, 213 fulfilled the screening criteria for pre-hospital notification; the AED was notified for 211 (99.1\%), in accordance with study protocol. Among patients for whom the AED was notified, $166(78.7 \%)$ were eventually diagnosed with acute stroke. The final diagnoses of patients without stroke included vasovagal syncope, fever/sepsis, and acute coronary syndrome (Fig 1).

Among the 211 patients for whom pre-arrival notification was performed, $32(15.2 \%)$ received reperfusion therapy (IVT and/or intra-arterial endovascular thrombectomy [IAT]). Reperfusion therapy was not administered to the remaining 134 patients for reasons such as intracranial haemorrhage, resolution of symptoms after arrival, and mild neurological deficits. Among the 85 patients who did not fulfil the screening criteria, one eventually received thrombectomy. Stroke notification was not performed for this patient due to uncertain time of symptom onset.

We compared the temporal trends in provision of acute stroke care after the implementation of ambulance stroke screening with a historical cohort from the period prior to implementation (Table 1). The door-to-CT time (25.6 min vs $32.0 \mathrm{~min}, \mathrm{P}=0.021$ ), door-to-needle time (49.2 $\mathrm{min}$ vs $70.1 \mathrm{~min}, \mathrm{P}=0.003$ ) [Fig 2], and door-to-groin puncture time for IAT (126.7 $\mathrm{min}$ vs $168.6 \mathrm{~min}, \mathrm{P}=0.04$ ) were significantly shortened after implementation of the pre-hospital stroke screening and notification system, compared with the historical control cohort. The proportions of patients who underwent CT within 25 minutes (68.6\% vs $51.6 \%, \mathrm{P}=0.001)$ and groin puncture within 120 minutes were also increased $(72.3 \%$ vs $18.2 \%, \mathrm{P}=0.008)$. Notably, the onset-to-door time was shortened by more than 1.5 hours (100.6 min vs $197.6 \mathrm{~min}, \mathrm{P}<0.001)$ after implementation of our system.

Furthermore, we compared the temporal trends in patients who received reperfusion therapy (ie, IVT and IAT) during the study period between patients who underwent pre-notification screening and those who did not (Table 2). The door-to-needle time was significantly reduced with pre-notification screening (49.2 $\mathrm{min}$ vs $75.2 \mathrm{~min}, \mathrm{P}=0.005$ ). The doorto-CT time and door-to-groin puncture time also tended to be shorter with pre-notification, although these differences were not statistically significant.

\section{Discussion}

The typical patient with stroke loses 1.9 million neurons per minute while the stroke remains untreated. ${ }^{7}$ Each minute saved between onset of stroke and treatment grants 1.8 days of extra healthy life. ${ }^{1}$ Timely reperfusion therapy with thrombolysis or thrombectomy has been demonstrated to reduce long-term disability when administered early to eligible patients. Earlier thrombolysis also lowers the risks of complications such as haemorrhagic transformation. ${ }^{8,9}$ Our study demonstrated that the implementation of a simple yet effective pre-hospital screening notification system significantly shortened time to diagnosis of acute stroke and subsequent intervention. This confirms the importance of prehospital notification in facilitating downstream management of patients with acute stroke by allowing the AED and stroke team to prepare for the patient's arrival in advance.

The efficiency of the acute stroke treatment pathway was improved by the notification system for the following reasons. First, early notification by the ambulance team allowed the AED and stroke 


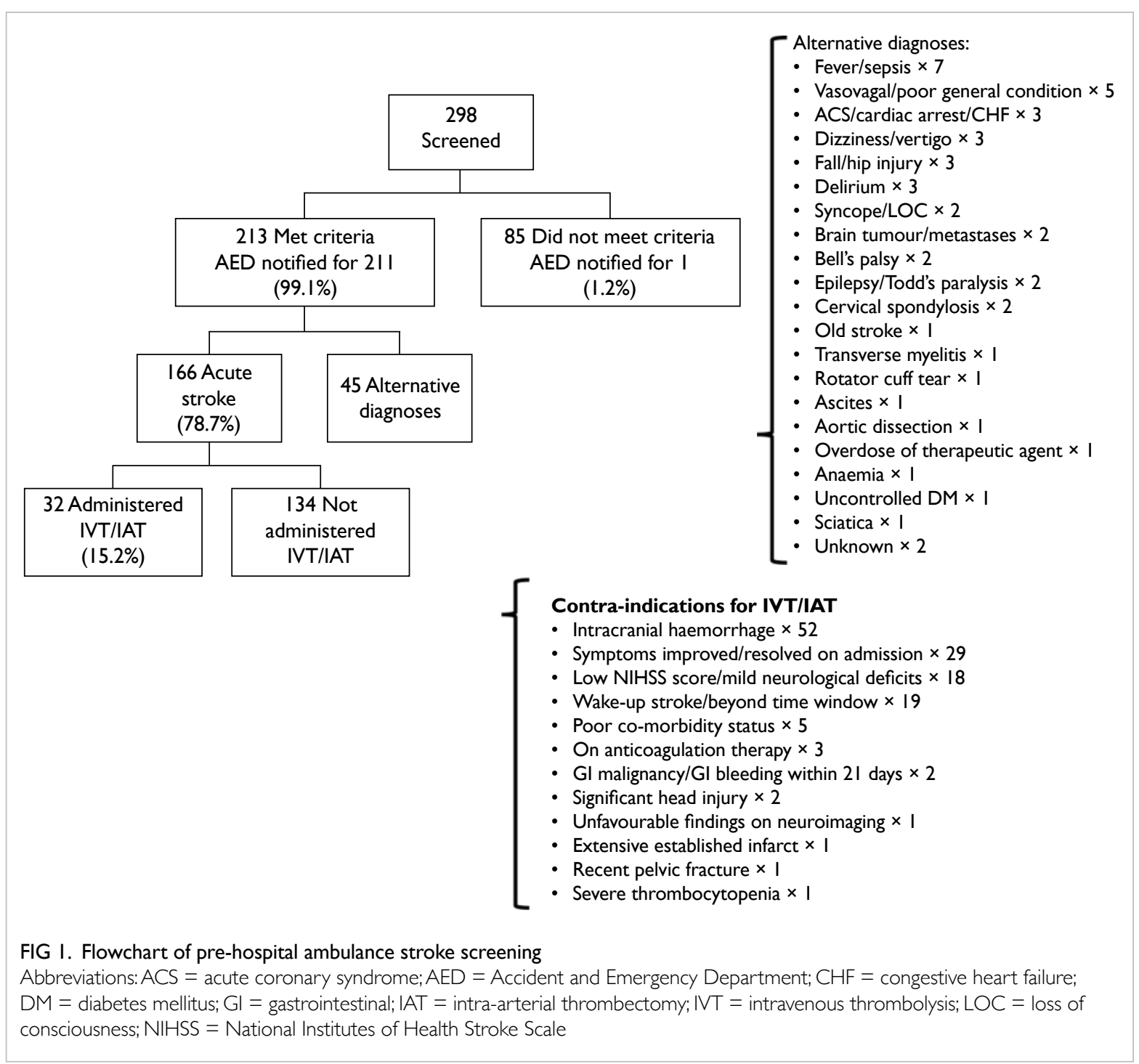

TABLE I. Demographic characteristics of acute ischaemic stroke patients and temporal trends affecting their treatment from January 2018 to October 2019*

\begin{tabular}{lccc}
\hline & $\begin{array}{c}\text { With notification, } \\
\text { Jul 2018 to Oct 2019 (n=166) }\end{array}$ & $\begin{array}{c}\text { Without notification, } \\
\text { Jan 2018 to Jun 2018 (n=318) }\end{array}$ & P value \\
\hline Sex (M:F) & $1.22: 1$ & $1.03: 1$ & 0.391 \\
Age, y & $71.5 \pm 14.8$ & $74.0 \pm 14.5$ & 0.071 \\
NIHSS score & $12.7 \pm 9.3$ & $12.6 \pm 10.3$ & 0.946 \\
\hline Onset-to-door time, min & $100.6 \pm 99.6$ & $197.6 \pm 295.0$ & $<0.001$ \\
Door-to-CT time, min & $25.6 \pm 27.8$ & $32.0 \pm 29.8$ & 0.021 \\
Door-to-CT <25 min & $114(69 \%)$ & $164(52 \%)$ & 0.001 \\
Patients receiving reperfusion therapy & $32(19 \%)$ & $70(22 \%)$ & 0.427 \\
Door-to-needle time, min & $49.2 \pm 16.7$ & $70.1 \pm 30.7$ & 0.003 \\
Door-to-needle time $<60$ min & $93(56 \%)$ & $122(38 \%)$ & 0.212 \\
Door-to-groin puncture time, min & $126.7 \pm 64.3$ & $168.6 \pm 73.9$ & 0.040 \\
Door-to-groin puncture time $<120$ min & $120(72 \%)$ & $58(18 \%)$ & 0.008 \\
\hline
\end{tabular}

Abbreviations: door-to-CT = Accident and Emergency Department arrival to brain computed tomography; NIHSS = National Institutes of Health Stroke Scale

* Data are presented as No. (\%) or mean \pm standard deviation, unless indicated otherwise 
physicians to assess the patient's medical history, including their eligibility and contra-indications for emergency reperfusion therapy, before patient arrival at the hospital. Second, the triage process at the AED was streamlined and patients with suspected stroke could be prioritised and immediately attended by the medical team. Moreover, the CT-suite staff were alerted to ensure the availability of a CT scan slot upon patient arrival. Third, the acute stroke nurse was consulted early to standby for patient arrival at the AED. Finally, the stroke alert created a sense of urgency among all medical and allied health professionals involved in stroke care, thereby enhancing the speed of the entire care pathway. Our experience is consistent with the reports from other medical systems that have implemented stroke alert systems to reduce door-to-needle time..$^{10-12}$

Notably, the present study demonstrated that the door-to-groin puncture time for thrombectomy candidates was also markedly reduced by pre-arrival notification, enabling the majority of patients to undergo groin puncture within 120 minutes. This is likely due to the longer time required to coordinate the neurointerventionist, interventional suite, and anaesthesiology care team; this process was initiated early with pre-arrival stroke notification. Consistent with our findings, pre-arrival notification is now recommended for all patients with suspected stroke, according to the 2019 guidelines of the American Stroke Association. ${ }^{3}$ Future efforts should focus on pre-hospital screening of thrombectomyeligible patients with large vessel occlusion and establishment of a diversion system to ensure patients are transported to thrombectomy-capable hospitals. ${ }^{10}$

One concern related to pre-hospital stroke screening was the potential for over-calling and thus overloading the acute stroke treatment pathway, thereby negatively affecting the AED service for patients with non-stroke emergencies. To maximise cost-effectiveness, the screening criteria

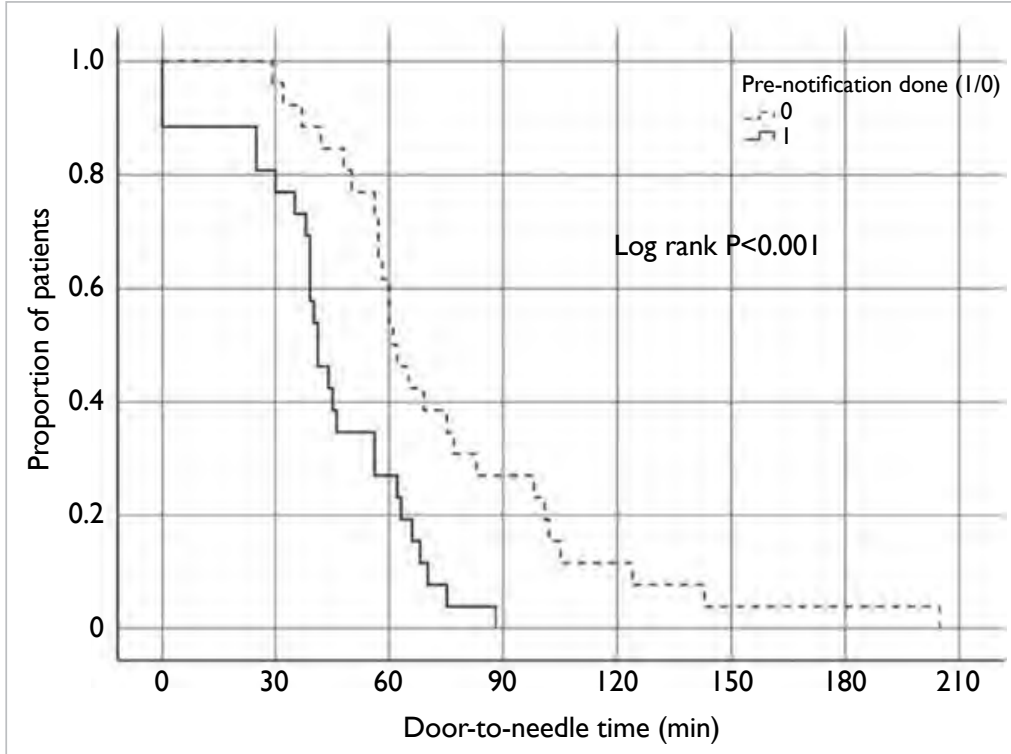

FIG 2. Kaplan-Meier curves of door-to-needle time with and without pre-hospital stroke screening and notification

were tailored to reduce notification for patients with stroke mimics and to exclude patients who were unlikely to benefit from acute reperfusion therapy. The addition of criteria such as the time of onset and pre-morbid functional status enabled efficient detection of reperfusion-eligible patients without excessively overloading the acute stroke treatment pathway. These approaches are known to prioritise patients with salvageable stroke for timely reperfusion therapy and are especially relevant in resource-limited public healthcare systems with restricted capacity to expeditiously manage all patients with stroke. ${ }^{13}$ As capacity improves, the criteria can be modified to include patients with delayed presentation after stroke onset.

TABLE 2. Temporal trends in provision of reperfusion therapy after implementation of pre-notification programme (Jul 2018 to Oct 2019)

\begin{tabular}{lccc}
\hline & $\begin{array}{c}\text { IVT/IAT with pre-notification } \\
(\mathbf{n}=\mathbf{3 2})\end{array}$ & $\begin{array}{c}\text { IVT/IAT without } \\
\text { pre-notification }(\mathbf{n}=\mathbf{3 6})\end{array}$ & P value \\
\hline Age, y & $74.3 \pm 13.2$ & $71.4 \pm 15.1$ & 0.38 \\
NIHSS score & $17.7 \pm 6.3$ & $18.9 \pm 9.4$ & 0.52 \\
Onset-to-door time, min & $79.4 \pm 64.2$ & $71.7 \pm 70.0$ & 0.85 \\
Door-to-CT time, min & $18.5 \pm 9.7$ & $23.4 \pm 13.5$ & 0.17 \\
Door-to-needle time (IVT), min & $49.2 \pm 16.7$ & $75.2 \pm 38.4$ & 0.005 \\
Door-to-groin puncture time (IAT), min & $(\mathrm{n}=21) 126.7 \pm 64.3$ & $(\mathrm{n}=20) 169.3 \pm 85.0$ & 0.12 \\
\hline
\end{tabular}

Abbreviations: door-to-CT = Accident and Emergency Department arrival to brain computed tomography; |AT = intra-arterial thrombectomy; IVT = intravenous thrombolysis; NIHSS = National Institutes of Health Stroke Scale

* Data are presented as mean \pm standard deviation, unless indicated otherwise 
Our study also validated the use of a Chinese version of the FAST criteria for screening of patients with suspected acute stroke. An important criticism of the FAST criteria is that they are not universally applicable in Chinese-speaking populations because there is no direct translation of "Face Arm Speech Time" to a memorable phase. ${ }^{14,15}$ In Hong Kong, the FAST mnemonic has been modified to “談笑用兵”, which is a well-recognised Chinese idiom where the characters represent Speech, Smile (Face), Mobilise (Arm), and Troop (Time to call for help). This version has been promoted widely by local healthcare professionals and stroke awareness organisations in the past decade. This concise screening algorithm is easily learned and rapidly performed, as reflected by the high accuracy in stroke detection by our ambulance staff after a 2-hour education session. To the best of our knowledge, this is the first study concerning the clinical utility of this modified screening algorithm. Recently, Chinese versions of similar scales, such as "Stroke 1-2-0" (China) and "Stroke 112" (Taiwan), have been proposed in other Chinese-speaking countries; each has achieved satisfactory acceptance among healthcare professionals..$^{14,15}$ However, these scales have not achieved widespread recognition by the general populations of those countries. Improved versions that aim to stratify large vessel occlusion strokes (ie, BE-FAST) or a new score developed specifically for use by ambulance staff may be introduced in the future. Comparative studies of these different scales and their applicability among Chinese-speaking countries will provide useful information to unify stroke awareness efforts across these populations. Further cost-effectiveness studies to evaluate the impact and sustainability of the system on a territory-wide scale will be beneficial for long-term infrastructure development in acute stroke care.

This study had some limitations. First, because of logistical considerations, symptom onset time of $\leq 4$ hours was used as a screening criterion. This was established on the basis of the practical expected times required to institute thrombolysis $(30 \mathrm{~min})$ or coordinate thrombectomy $(120 \mathrm{~min})$ at the time of study initiation. Because the time window for thrombectomy has been expanded from 6 to 24 hours in patients with favourable penumbra demonstrated with CT perfusion imaging, the cut-off time of symptom onset can be extended accordingly. Second, EMS personnel may serve multiple catchment areas. Hence, patients with stroke may be transported by ambulances with EMS personnel who did not undergo the additional training; these patients may not be screened and hospitals may not be notified before patient arrival. Third, clinical outcome data were not consistently available for the historical cohort, precluding analysis of clinical benefits due to improved stroke treatment times. This issue might be resolved through territory-wide adoption of a stroke screening protocol.

\section{Conclusion}

Implementation of pre-hospital stroke screening using criteria based on a modified version of the FAST test, together with pre-arrival notification, significantly shortened the door-to-reperfusion therapy time for patients with acute ischaemic stroke. Pre-hospital stroke screening during ambulance transport by EMS personnel who complete a 2-hour focused training session is effective for identifying reperfusion-eligible patients with stroke.

\section{Author contributions}

Concept or design: KC Teo, MMY Tse, WM Lui, TC Tsang, ACO Tsang.

Acquisition of data: WCY Leung, WM Kwok, LHC Lam, OMY Choi, ACO Tsang.

Analysis or interpretation of data: All authors.

Drafting of the manuscript: WCY Leung, KC Teo, OMY Choi, ACO Tsang.

Critical revision of the manuscript for important intellectual content: All authors.

All authors had full access to the data, contributed to the study, approved the final version for publication, and take responsibility for its accuracy and integrity.

\section{Conflicts of interest}

All authors have no conflicts of interest to disclose.

\section{Acknowledgement}

We would like to acknowledge the ambulance staff of the Ambulance Command (Hong Kong Division), Fire Services Department for the support in this project and in improving stroke patient care in Hong Kong.

\section{Funding/support}

This research received no specific grant from any funding agency in the public, commercial, or not-for-profit sectors.

\section{Ethics approval}

The study was approved by The University of Hong Kong/ Hospital Authority Hong Kong West Cluster Research Ethics Committee (Ref UW20-442). The patients provided written informed consent for all treatments and procedures.

\section{References}

1. Meretoja A, Keshtkaran M, Saver JL, et al. Stroke thrombolysis: save a minute, save a day. Stroke 2014;45:1053-8.

2. Adeoye O, Nyström KV, Yavagal DR, et al. Recommendations for the establishment of stroke systems of care: a 2019 update. Stroke 2019;50:e187-210.

3. Powers WJ, Rabinstein AA, Ackerson T, et al. Guidelines for the early management of patients with acute ischemic stroke: 2019 update to the 2018 guidelines for the early management of acute ischemic stroke: a guideline for 
healthcare professionals from the American Heart Association/American Stroke Association. Stroke 2019;50:e344-418.

4. Tsang AC, Yeung RW, Tse MM, Lee R, Lui WM. Emergency thrombectomy for acute ischaemic stroke: current evidence, international guidelines, and local clinical practice. Hong Kong Med J 2018;24:73-80.

5. Kidwell CS, Starkman S, Eckstein M, Weems K, Saver JL. Identifying stroke in the field. Prospective validation of the Los Angeles prehospital stroke screen (LAPSS). Stroke 2000;31:71-6.

6. Harbison J, Hossain O, Jenkinson D, Davis J, Louw SJ, Ford GA. Diagnostic accuracy of stroke referrals from primary care, emergency room physicians, and ambulance staff using the face arm speech test. Stroke 2003;34:71-6.

7. Saver JL. Time is brain-quantified. Stroke 2006;37:263-6.

8. Saver JL, Fonarow GC, Smith EE, et al. Time to treatment with intravenous tissue plasminogen activator and outcome from acute ischemic stroke. JAMA 2013;309:2480-8.

9. Lees KR, Bluhmki E, von Kummer $R$, et al. Time to treatment with intravenous alteplase and outcome in stroke: an updated pooled analysis of ECASS, ATLANTIS,
NINDS, and EPITHET trials. Lancet 2010;375:1695-703.

10. Chiu YC, Tang SC, Sun JT, et al. Using G-FAST to recognize emergent large vessel occlusion: a training program for a prehospital bypass strategy. J Neurointerv Surg 2020;12:104-8.

11. Tan BY, Ngiam NJ, Sunny S, et al. Improvement in doorto-needle time in patients with acute ischemic stroke via a simple stroke activation protocol. J Stroke Cerebrovasc Dis 2018;27:1539-45.

12. Zhang S, Zhang J, Zhang M, et al. Prehospital notification procedure improves stroke outcome by shortening onset to needle time in Chinese urban area. Aging Dis 2018;9:42634.

13. Tsang AC, Yang IH, Orru E, et al. Overview of endovascular thrombectomy accessibility gap for acute ischemic stroke in Asia: a multi-national survey. Int J Stroke 2020;15:51620.

14. Zhao J, Eckenhoff MF, Sun WZ, Liu R. Stroke 112: a universal stroke awareness program to reduce language and response barriers. Stroke 2018;49:1766-9.

15. Zhao J, Liu R. Stroke 1-2-0: a rapid response programme for stroke in China. Lancet Neurol 2017;16:27-8. 